\title{
Tropes, causal processes and functional laws
}

\author{
Markku Keinänen \\ University of Turku \\ [DRAFT, please do not quote]
}

\begin{abstract}
In this article, I start with the assumption that all fundamental monadic properties dispositional quantity tropes. I argue that the dispositional tropes and the causal processes they produce can, in relevant part, account for the truth of causal functional laws (such as Coulomb law): laws of nature that describe the forces the quantitative properties falling under a determinable generate as a function of their distance. Following Ellis \& Lierse (1994) and Ellis (2001), I adopt the claim that a large group of the dispositional properties figuring in functional laws are causal powers and that their manifestations are causal processes. The forces (gravitational force, Coulomb force) resulting from these causal processes are connected by the proportion relations in accordance with the formal proportion relations between the tropes producing the processes. Hence, property tropes and the respective causal processes suffice to secure that the resulting forces accord with the functional formula.
\end{abstract}

\section{Introduction}

According to the dispositionalist conception of natural properties, all fundamental intrinsic natural properties are dispositional: necessarily, if object $a$ instantiates intrinsic property $\mathrm{F}$, property $\mathrm{F}$ bestows on $a$ with certain definite causal powers to act in certain ways in certain kinds of circumstances. Two related observations give a strong support for the dispositionalist conception of properties: first, it seems that all empirically discovered fundamental intrinsic properties (e.g., masses, electric charges, spin quantum numbers, quark colour charges) are physical quantities and dispositional properties of objects (Ellis 2001, 115; Mumford 2006). The bearers of these properties (i.e., fundamental physical microparticles such as electrons and quarks) are powerful particulars, i.e., all of their intrinsic properties are dispositional. Second, we identify the fundamental properties (such as masses and charges) by means of the laws of nature (Newton's law of gravitation, Coulomb's law), which describe the associated causal powers, or, the behaviour of objects having these properties in different kinds of circumstances. If we maintain that some radically different laws of nature could be true of these properties or that the same laws could describe the 
causal powers associated with quite different properties, it is difficult say of what properties we are talking about. ${ }^{1}$

Hence, the dispositionalism about fundamental properties (in some form) accords with the empirical ways the fundamental properties are identified (by means of the associated causal powers the laws of nature describe). ${ }^{2}$ According to certain prominent recent dispositionalists (Ellis 2001; Mumford 2004; Bird 2007), dispositional properties are also sufficient truthmakers for the functional laws describing the associated causal powers. We need not introduce any further entities such as relations between universals to make them true. As a consequence, certain dispositionalist theories claim to have an ontological benefit of accounting for the truth of functional laws without introducing such contestable entities as laws or relations between universals (cf. Mumford 2004; Yates 2012: sec. 2.2).

Most of the leading recent dispositionalists are advocates of property universals. They have formulated their accounts either exclusively by means of Russellian property universals (Bird 2007) or by means of tropes and Neo-aristotelian property kind universals (Ellis 2001). The tropes vs. universals debate has been considered independent of the dispositionalism vs. categoricalism debate. Alternatively, since the laws of nature seem to make claims about property universals, universals might be considered to have an advantage over tropes.

In this article, I argue that the dispositional tropes and the causal processes they produce can, in relevant part, account for the truth of causal functional laws (such as Coulomb law): laws of nature that describe the forces the quantitative properties falling under a determinable generate as a function of their distance. I adopt the trope theory SNT of powerful particulars, which I have defended elsewhere (Keinänen 2011). In section 3, I explain why functional laws are prima facie problematic for the trope theorist. I adopt the claim that a large group of the dispositional properties figuring in functional laws are causal powers and that their manifestations are causal processes (cf. Ellis \& Lierse 1994 and Ellis 2001) in order to deal with these difficulties (section 4). To get these arguments off the ground, I give a short of overview of the trope theory SNT and the resemblance of quantity tropes, which is the topic of the next section.

\footnotetext{
${ }^{1}$ Yates (2012: sec. 2.1) gives an excellent overview of the difficulties a "quidditist" believer of the possibility of radically different laws of nature faces with respect to the identification of properties.

${ }^{2}$ Dispositionalism a wide sense covers both (strong) "dispositional essentialism", according to which laws of nature are metaphysically necessary (Ellis \& Lierse 1994; Ellis 2001; Bird 2007) and weaker positions such as Hendry's \& Rowbottom's (2009) “weak dispositional essentialism”, according to which laws could have been slightly different.
} 


\section{Quantity tropes: the basic constituents of objects}

Tropes are concrete (i.e., spatio-temporal or, at least, temporal) particulars. ${ }^{3}$ Most of the alternative trope theories (i.e., trope bundle theories of substance) agree in certain main features of property tropes. Tropes have the paradigmatic category features of fundamental concrete particulars. First, they are particular: the distinct tropes are capable of being intrinsically exactly similar. ${ }^{4}$ As spatio-temporal entities tropes usually have a single, possibly extended, spatio-temporal location. Second, they are countable individuals, i.e., they have certain determinate identity conditions and are countable. ${ }^{5}$ As a consequence, any trope is distinct from all other individuals. Third, as fundamental entities tropes are categorially simple: every trope is either simple or it is composed of further property tropes. Objects (or, substances) are certain kinds of aggregates of property tropes. Finally, tropes have certain determinate identity conditions independent of the identity conditions of any other entity; they are identity independent existents. ${ }^{6}$

Moreover, tropes are particular properties: they have two further features in addition to the standard category features of fundamental concrete particulars. First, property tropes can exist as spatio-temporally co-located (or, compresent) with each other. Since some simple substances (i.e. powerful particulars) might also be capable of co-location, this does not count as a distinctive feature of tropes. Rather, it is distinctive to tropes that they always occur as co-located with the other tropes. Second and more importantly, tropes have a thin particular nature to determine a single feature of the object possessing the trope (Campbell 1990: 5960; Simons 2003: sec.6). It is customary to identify tropes with the natures they determine. For instance, a $1 \mathrm{~kg}$ trope of object $a$ might be considered as that particular mass of object $a$. Nevertheless, tropes are entities with the above category features and cannot be identified

\footnotetext{
${ }^{3}$ Certain trope theorists (such as Williams 1953: 7-8; Campbell 1990: 53-56 and Denkel 1996) allow of the possibility of non-spatial (e.g., purely temporal) tropes. However, since it is contestable whether there can be such tropes, I will not consider them further here.

${ }^{4}$ Cf. Williams (1986) and Ehring (2011), for a defence of this account of particularity.

${ }^{5}$ Lowe (1998: ch.3) calls entities with both determinate identity conditions and countability individual objects. In different categorial schemes, these category features (e.g., particularity, determinate identity conditions and countability) can vary independently of each other. Thus, for instance, universals might be considered as countable individual objects or non-countable entities with determinate identity conditions.

${ }^{6}$ Most of the recent trope theorists consider the individuation of tropes as primitive, cf., e.g., Campbell (1990); Ehring (2011) and Keinänen \& Hakkarainen (2013). Schaffer (2001) individuates tropes by their spatiotemporal location, which seems to conflict with their identity independence.
} 
with anything less determinate (such as "natures"). Therefore, I am confined to claiming that the nature of $1 \mathrm{~kg}$ trope $t$ of object $a$ is to determine the mass of object $a$ having $t$ as a certain kind of proper part. ${ }^{7}$

Although mainly agreeing about the above category of features of tropes, the alternative trope theories (i.e., trope bundle theories of substance) differ in the construction of objects (or, substances). According to the trope theory SNT (Keinänen 2011: sec. 4), only simple substances (powerful particulars) trope bundles, whilst complex objects are constituted by simpler objects. The SNT divides the tropes constituting an object into two distinct groups. First, it introduces one or more nuclear tropes rigidly dependent on each other to determine the necessary features of a powerful particular (such as a down quark or an electron). ${ }^{8}$ The distinct nuclear tropes of substance must fall under distinct determinables (mass, charge, etc.). Second, many simple substances must have contingent tropes, which are one-sidedly rigidly dependent on the nuclear tropes. Property trope $t$ is a part of substance $i$ if and only if $t$ is rigidly dependent only on its nuclear tropes. Simple substances are trope aggregates in which all of the rigid dependencies of the member tropes are fulfilled.

The most important distinctive feature of the SNT relative to Simons' (1994) nuclear theory, from which it is developed, is to provide a comprehensive account of location of tropes. The main idea is that tropes are not independently located entities. Rather, their location is determined by the location of the trope bundles in which they occur. Here, suffices it to consider the location of nuclear tropes. According to the SNT, the mutually rigidly dependent nuclear tropes of substance $i$ form an individual (the $n$-bundle) that is in the basic spatio-temporal relations determining the location of the $n$-bundle. Since the $n$-bundle is a minimal entity occurring in the basic spatio-temporal relations, its location determines the location of the nuclear tropes. Nuclear tropes must have some location and their existence entails the existence of the $n$-bundle. Consequently, the existence of nuclear tropes also entails that they are co-located (Keinänen 2011: 438-440).

Further, the location of the nuclear tropes determines the centre of influence (spatiotemporal location) of a powerful particular of which they are parts. Elementary fermions constitute the best a posteriori examples of powerful particulars. For instance, it seems that a down quark has a mass trope of $4.8 \mathrm{MeV}$, charge trope of $-1 / 3 \mathrm{e}$ and spin quantum number

\footnotetext{
${ }^{7}$ The different trope theories analyze inherence (i.e., the relation between a property trope and the object possessing the trope) in different ways, cf. Keinänen (2011: 429ff.) for an overview.

${ }^{8}$ Let " $\leq$ " be a relation of improper parthood between distinct entities (cf. Simons (1987: 112) for the definition) and "E!" the predicate of (singular) existence. Trope $e$ is strongly rigidly dependent on trope $f$, if $\operatorname{SRD}(\mathrm{e}, \mathrm{f}) \equiv$ $\neg(\square \mathrm{E} ! \mathrm{f}) \wedge \square((\mathrm{E} ! \mathrm{e} \rightarrow \mathrm{E} ! \mathrm{f}) \wedge \neg(\mathrm{f} \leq \mathrm{e}))$ holds.
} 
trope of $1 / 2$ in its trope nucleus. Moreover, the SNT allows of a powerful particular to have some contingent tropes. Colour charge tropes of a quark might be good candidates.

The standard way to describe the resemblance of tropes is by means of primitive internal relation of exact similarity or less than exact resemblance. ${ }^{9}$ According to the SNT, all tropes are quantities. Therefore, I propose a more accurate description of their "resemblances" in terms of the different formal relations of proportion and the relation of order. ${ }^{10}$ For instance, $1 \mathrm{~kg}$ trope $t$ is in formal relation of $1: 1$ proportion to any other $1 \mathrm{~kg}$ trope. Similarly, all and only mass tropes are mutually connected by some formal relation of proportion; the tropes falling under a distinct determinable (e.g., charge tropes) do not bear the relations of proportion to any mass trope. Moreover, all mass tropes mutually are connected by the formal relation of order, i.e., "equal or greater than", which explains the asymmetry of proportion relations (1: 1 proportions excluded). Trope $t$ is a mass trope if and only if $t$ bears some relation of proportion and the relation of order to any mass trope.

The proposed account seems to be deal with the "resemblances" between most of the basic physical quantities (such as electric charge, spin, and space-time interval) if we keep in mind that many of them (e.g., charges) can have both positive and negative values. ${ }^{11}$ In such case, the relations of proportion also take positive and negative values. Although the proportions between quantity tropes are not conventional, the choice of the quantitative unit is. $^{12}$

Why are we entitled to consider the relations of proportion and order as formal ontological relations? Why don't we need to introduce further entities, e.g., the relational tropes of proportion to account for the proportions between distinct tropes? Here, I am confined to pointing out that the relations of proportion between tropes have two general characteristics of a formal ontological relation and are plausibly considered as formal relations. ${ }^{13}$ First, assuming that all tropes are quantities, every trope must be connected to certain other quantity tropes by some proportion relation. It must have a specific position in a

\footnotetext{
${ }^{9}$ Cf. Campbell (1990: 36ff); Maurin (2002); Simons (2003: sec.6).

${ }^{10}$ The present suggestion to employ the relations of proportion to spell out the relations between determinate quantities is inspired by Bigelow \& Pargetter's (1990: 55-62) theory. Bigelow \& Pargetter introduce proportions as second-degree relation universals, i.e., relations between relation universals. In the present approach, the proportion relations are treated as formal relations, cf. below.

${ }^{11}$ However, we need to develop the approach further in order to have a similar account of the necessary relations between the tropes of quark colour charge. Moreover, we need further work to generalize the present approach to the tropes determining distances in different directions and to specify the formal relations between them.

${ }^{12}$ When we introduce the unit of electric charge, we have a freedom to choose which of the values of charge are negative and which positive if we keep the positive and negative proportions as fixed.

${ }^{13}$ For more discussion of formal relations, cf. Smith \& Mulligan (1983); Smith (1998); Correia \& Keller (2004) and the other articles in the same issue of Dialectica.
} 
network formed by the distinct proportion relations. Hence, as do mereological relations and the relations of existential dependence, the relations of proportion form a network and the existence of a trope presupposes that it has some specific position in the different networks of these different formal relations.

Second, since the existence of trope $t$ presupposes that we can specify its proportion relations to the other tropes, the relations of proportion (as are the other formal relations) are ungrounded internal relations, i.e., they obtain because the related entities exist. Consider, for instance, the relation of 3:1 proportion between -e charge trope $t_{1}$ and -e/3 charge trope of $t_{2}$. Necessarily, if $t_{1}$ and $t_{2}$ exist, they are in the relation 3:1 proportion to each other. Further, because $t_{1}$ and $t_{2}$ are the tropes they are, i.e., they have their specific thin nature, they are connected by the relation 3:1 proportion. In similar way, we can argue that the relations of order are formal relations.

Since formal relations are ungrounded internal relations, they are not relational entities but rather relational predications made true by the entities related; instead of calling them formal relations, we could call them formal ontological predications. We can go even further and identify determinate and determinable kinds of tropes with certain kind terms applying to tropes. Here, suffices it to point out that given trope $t$ belongs to certain determinate kind D, e.g., is a $1 \mathrm{~kg}$ trope because it is the trope it is and bears the relation of $1: 1$ proportion to any $1 \mathrm{~kg}$ trope. Consequently, trope $t$ is a (minimal) truthmaker of the claim that $t$ is a $1 \mathrm{~kg}$ trope. Similarly, trope $t$ suffices to make true the proposition that $t$ is a mass trope.

\section{Functional laws}

Functional laws (such as Newton's inverse square law of gravitation and Coulomb's law) spell out relations between determinate quantities falling under some distinct determinables (e.g., masses, distances and gravitational attractions by some force). In expressing these relations, they are completely indiscriminative between the distinct determinates falling under a determinable: if true, a functional law holds true for every determinate value of each quantity it contains.

Given that (at least some) functional laws are true law statements, they pose a prima facie difficulty for the trope nominalist. Let us call a law statement expressing a relation between (the most specific) determinates falling certain determinables a determinate law 
(e.g., Newton's law of gravitation for some specific masses and some specific distance). The truth of the functional law ("determinable law") entails that a prima facie infinite number of determinate laws are true. The first difficulty results from the observation that the determinate laws are logically independent of each other. Since the determinate laws are mutually logically independent, the co-ordination of the distinct determinate laws (that they fall under the same functional formula, the determinable law) seems to need for explanation. Second, if determinable quantities such as mass have uninstantiated but possibly instantiated values, the functional law entails truths about uninstantiated but possibly instantiated determinate quantities (such as masses). Prima facie, these truths need to have a truthmaker (cf. Armstrong 1997: 243-248). Before spelling out of why these are difficulties for the trope nominalist, we must specify some principles of truthmaking and how the truth of determinate laws depends on what exists.

Certain leading dispositionalists (e.g., Ellis 2001; Mumford 2004) maintain that dispositional properties are sufficient truthmakers for functional laws such as Newton's law of gravitation or Coulomb's law. Intuitively, these laws are just law statements whose truth is grounded by the existence of dispositional properties. A more explicit expression of this idea requires that we specify the principles of truthmaking we presuppose. Here, I am confined to assuming two standard principles of truthmaking: first, truthmakers are entities of any category and the items made true are (atomic) propositions (truth-bearers). Second, the existence of truthmaker(s) entails the truth of the proposition made true. If a group of entities makes proposition $p$ jointly true, then the existence of all of the entities belonging to the group entails that $p$ is true. ${ }^{14}$

Two distinct dispositional properties $D_{1}$ and $D_{2}$ (two distinct determinate mass universals) are not sufficient truthmakers for the determinate law concerning them. Rather, it seems that all instantiations of these dispositional properties (mass universals) in certain distance from each other constitute a sufficient truthmaker. For instance, one might maintain that the instantiations of two distinct values of mass, say $M_{1} \mathrm{x}$ and $M_{2} \mathrm{y}$ and the instantiations of the dyadic relation of distance Rxy by all objects $x$ and $y$ having these masses in distance $\mathrm{R}$ from each other make jointly true the determinate law of gravitation concerning masses $M_{1}$ and $M_{2}$ in distance $\mathrm{R}$.

\footnotetext{
${ }^{14}$ A group (or, a plurality) of entities exists if and only if all its members exist. Certain entities make jointly true proposition $p$ if and only if their plurality makes $p$ true. I adopt the following familiar entailment principle for joint truthmaking: if a plurality of entities makes a proposition $p$ true, then the existence of the plurality (and all members of the plurality) entails that $p$ is true, cf. Mulligan et al. (1984: sec. 6).
} 
Unfortunately, this is not quite right. Truthmakers are entities (existent beings) whose existence is supposed to entail the truth made true. On the above account, the individual instances of a determinate law of gravitation are made true by the pairs of objects instantiating the determinate masses and distance relation $R$. Nevertheless, all objects having these properties in a certain distance from each other that actually obey a determinate law of gravitation do not jointly make the determinate law true for their existence does not entail that all pairs of objects that fulfil the same condition obey the determinate law. Since the trope theorist cannot postulate general facts, we must reject the demand that determinate laws are made true. For the present purposes, suffices it to maintain that a determinate law is true because it does not have falsifying instances: if two arbitrary objects $x$ and $y$ fulfil the antecedent condition (i.e., have masses $M_{1}$ and $M_{2}$ in distance R), they also fulfil the consequent condition (attract each other by certain force $\mathrm{F}$ ). ${ }^{15}$

Assume now that properties and relations are tropes instead of universals. The situation changes as follows: an instance of the corresponding law is made true by mass tropes $t_{1}$ (of value $M_{1}$ ), $t_{2}$ (of value $M_{2}$ ) and by relational trope $r$ (of distance $R$ ) determining the distance between tropes $t_{1}$ and $t_{2}{ }^{16}$ The determinate law is true because it does not have falsifying instances. Given that two objects having dispositional tropes falling under these determinates exist and are in certain distance $\mathrm{R}$ from each other, they attract each other by certain force F. Thus, on this view, the existence of the property tropes and relational tropes at issue entails that the instances of the corresponding determinate law are true: necessarily, if the property and relational tropes exist, the instances of the determinate law hold true. There is no room for considering, e.g., the value of natural constant associated with the law (such as gravitational constant or Coulomb constant) as metaphysically contingent. ${ }^{17}$

The dispositionalist trope nominalist can now suggest a generalization of this strategy to ground the truth of functional laws: since the determinate laws falling under a functional law do not have counter-instances, also the functional law is true. Nevertheless, the two prima facie difficulties discussed above seem to block this suggestion:

\footnotetext{
${ }^{15}$ Yates (2012: sec. 3.3) uses a similar technique to ground the laws of nature by using Bird's (2007) conditional analysis of dispositions. However, according to his approach, dispositional properties are equivalent to conjunctions of stimulus manifestation conditionals. No manifestation partners are introduced.

${ }^{16}$ I here make a simplifying assumption that the distances between tropes are accounted for by the relational tropes of distance between the trope bundles having the tropes as their parts, although there are other alternatives: e.g., that they are grounded by the relational tropes of occupation between tropes and the regions of space-time.

${ }^{17}$ Hence, "weak dispositional essentialism" in any of its forms is ruled out, cf. note 2.
} 
Governing function: we must introduce some entity or entities that ontologically ground the co-ordination of the distinct determinate laws falling under a single functional formula (functional law).

Since the determinate laws are logically independent of each other, it seems that their truthmakers cannot make true (or, ontologically ground) the further claim that the determinate laws fall under a single functional formula, i.e., are co-ordinated. While realists might introduce determinable universals to ground this claim, the trope nominalist does not have such further ontological resources at her disposal. ${ }^{18}$

Second, and even more seriously, the truthmakers of the instances of determinate laws (existent determinate property and relation tropes) do not make true the instances of determinate laws about the possible but uninstantiated determinate quantities (e.g., specific uninstantiated masses). Since functional laws (e.g., Newton's law of gravitation) seem to entail such propositions, we need to introduce some further entities to make them true:

Guaranteeing function: we must postulate some entity or entities that ontologically ground the fact that the true functional law (e.g., Newton's law of gravitation) is true of every (instantiated or uninstantiated) possible value of the quantities it contains.

Again, it seems to be a natural choice to introduce determinable universals to make the instances of a functional law true.

By the same token, it seems that the resources of the present approach are seriously limited. While characterizing dispositional property tropes, we are able to rely on contextual claims on them that are not consequences of their role as truthmakers of the instances of a determinate law. For instance, we are entitled to maintain that a determinate law describing the behaviour of the tropes belonging to exactly the same determinate kinds does not have any falsifying instances. Similarly, it is fairly unproblematic to maintain that if the distinct determinate dispositional tropes fall under a single determinable, the distinct determinate laws describing their behaviour fall under a single functional formula. What is the missing element?

\footnotetext{
${ }^{18}$ Armstrong (1997) introduces determinable universals to ground functional laws. Ellis (2001: sec. 2) postulates determinable universals (considered as kinds of tropes) for the more general purpose of accounting for the division of tropes into determinable kinds.
} 


\section{Tropes and causal processes}

In this section, I argue that the missing element is causal processes. Given that we provide a correct description of the kinds of causal processes certain kinds of dispositional property tropes generate, we are able to understand why their behaviour can be described with the help of a functional formula. Unlike many standard approaches, the present approach does not rely on the stimulus manifestation conditionals in the description of the "essences" of dispositional properties. Rather, on the present approach, dispositional properties are best classified on the basis of their characteristic effects. ${ }^{19}$ In general level, Ellis (2001: 128) makes a useful three-fold distinction between propensities (dispositions to behave in a certain way in any of the wide range of circumstances: e.g., a propensity of a radium atom to decay in certain time in a certain way), capacities (dispositions distinguishable by the kinds of consequent events they are able to produce, e.g., inertial mass) and causal powers (dispositions to produce forces of a certain kind, e.g., gravitational mass). Ellis maintains that the fundamental properties are dispositions of some of these basic kinds. I leave open the question of whether the number of different kinds of fundamental dispositions can still be reduced.

Here, I am confined to describing the role of causal powers in connection with causal processes and leave the other kinds of dispositions to some other occasion. Salient examples of causal powers functional laws characterize are gravitational masses and electric charges. An object possessing a trope of a determinate kind of causal power (e.g., -e charge trope $t_{1}$ ) produces an attractive or a repulsive force on an object possessing another trope of the same determinable kind (e.g., e charge trope $t_{2}$ ). We can refer to the manifestations of causal powers by means of the traditional terminology of forces: e.g., gravitational attraction by force $F$ to a certain direction. Nevertheless, instead of relying on primitive forces, the current quantum physics describes the manifestations as energy-transfer processes constituted by the exchange of the "force-carrier" bosons (such as gluons and photons). ${ }^{20}$

Hence, I assume that the manifestations of causal powers are causal processes. ${ }^{21}$ They involve collections of physically primitive entities (virtual bosons), which are emitted by a

\footnotetext{
${ }^{19} \mathrm{Cf}$. Ellis 2001: $123 \mathrm{ff}$. For instance, the characteristic effects of causal powers are the causal processes they produce.

${ }^{20} \mathrm{Cf}$. Griffiths (1987) for a useful introduction into physical theories (quantum electrodynamics and quantum chromodynamics) about these processes.

${ }^{21}$ Cf. Ellis \& Lierse (1994); Ellis (2001) and Handfield (2010). Both Ellis and Handfield assume that the manifestations of all dispositional properties are processes (of some general type) that divide into natural kinds. However, I will not discuss these further assumptions here.
} 
fermion and exist until some fermion absorbs them. It would be tempting to identify these basic causal processes with (collections of) their mediators but the exact categorization of these entities would require a closer look at the physical reality. ${ }^{22}$

In many cases, quantity tropes (e.g., -e charge tropes) belonging to a determinate kind produce these processes in presence of "manifestation partners", which are further tropes falling under the same determinable (charge tropes) in a certain distance from the first tropes. The laws of action describing the generation of causal processes by the tropes falling under a single determinable are functional laws (such as Coulomb's law or Newton's gravitation law). Any two tropes generating a causal process are mutually connected by some relation of proportion (they belong to the same determinable kind). Moreover, if the relevant functional law is true, the forces that result from the causal processes the different kinds of tropes falling under the same determinable produce are mutually connected by the relations of proportion in accordance with the proportion relations between the tropes.

Take, e.g., the scalar form of Coulomb's law, where $\mathrm{q}_{1}$ and $\mathrm{q}_{2}$ are different electric charges, $k_{\mathrm{e}}$ is the Coulomb constant and $|F|$ the absolute value of the resulting electrostatic force:

$$
|\boldsymbol{F}|=k_{e} \frac{\left|q_{1} q_{2}\right|}{r^{2}}
$$

The force $\boldsymbol{F}$ associated with a causal process produced by the charge tropes of different determinate kinds (determining $\mathrm{q}_{1}$ and $\mathrm{q}_{2}$ ) varies in accordance with the proportion relations between the charge tropes if distance $r$ remains constant. Consequently, the dispositional property tropes and causal processes take care of the governing function, i.e., the coordination of determinate laws under a single functional law. ${ }^{23}$ We need not introduce any further entities. Similarly, because the tropes belonging to the same determinate kind (i.e., tropes connected by 1:1 proportion) produce causal processes with resulting forces in 1:1 proportions to each other, determinate laws do not have any falsifying instances.

\footnotetext{
${ }^{22}$ Morganti (2009) proposes a unified account of all elementary particles of the Standard Model as trope bundles. However, because virtual bosons have process-like features and an existentially dependent status, it is highly questionable whether they should be treated as any kinds of objects. Thus, it seems that the trope bundle theory of bosons still needs to be worked out.

${ }^{23}$ Similarly, after arguing against the view that laws of nature could be considered to have a genuine governing role (or, the role of determining the course of events given certain initial conditions), Mumford (2004: sec. 11) assigns the governing role to dispositional properties.
} 
It seems that the force necessarily associated with a causal process is not further dispositional property but rather determined by the structure of the corresponding causal process. However, the more exact characterization of the determination would require a quantum mechanical characterization of the process. On the present approach, the truthmakers of the instances of a determinate law are tropes and the causal processes they produce. Thus, unlike the dispositionalist conception of truthmaking described in the previous section, the present approach is not committed to the claim that the determinate laws of action are metaphysically necessary. Nor does it need to reject the claim. It is consistent with the present approach that the value of a natural constant associated with a functional law (e.g., Coulomb constant) is metaphysically contingent and that all dispositional tropes of a determinable kind (e.g., charge tropes) could have produced causal processes in a slightly different way.

Nevertheless, I have not yet dealt with the guaranteeing function: introduced an entity or entities to ground the truth of a functional law for the possible but uninstantiated values of the dispositional properties. Let e/6 be such value of electric charge (the physical inaccuracy of this example does not matter because there might well be possible but uninstantiated values of some other dispositional properties, e.g., masses). If true, Coulomb law predicts that dispositional e/6 charge trope $t_{1}$ would, in presence of trope $t_{2}$ of positive unit charge e in a fixed distance from $t_{1}$, produce electrostatic repulsion $1 / 2$ times of the repulsion produced by e/3 charge trope $t_{3}$ and unit charge trope $t_{2}$.

On the present account, dispositional tropes are (partly) identified by the kinds of causal processes they produce. I use the term "identification" here for individuation in epistemic sense, i.e., how we are able to actively identify tropes as non-identical with the other tropes. ${ }^{24}$ By contrast, I suggest to take the individuation of tropes as primitive and reject the further claim that the kinds of causal processes a trope produces individuate it (in metaphysical sense). Since trope $t$ has its nature independently of the existence of the other tropes, the network of proportion relations in which trope $t$ is does not individuate it either. However, we can use these proportion relations to help the identification of trope $t$.

Consider now possible e/6 charge trope $t_{1}$ taken up above. We would have some clear ways to identify trope $t_{1}$ (as a property of a certain kind of object and a trope that would produce certain kinds of effects in presence of certain existent tropes). Therefore, the

\footnotetext{
${ }^{24}$ Cf. Keinänen \& Hakkarainen (2013: sec. 1) for the distinction between identification and individuation in metaphysical sense. We argue that tropes are identified (in epistemic sense) as properties of certain kinds of substances (op cit.: sec. 4). Moreover, tropes are identified by their location and nature together with the kinds of causal processes they produce.
} 
existence of $t_{1}$ seems to be conceivable. By contrast, it is not clear whether the predictions Coulomb law makes about the behaviour of $t_{1}$ are true. Compare them with the counterfactual claims about the behaviour of actual property tropes. Assume that two powerful particulars instantiate a positive unit charge (the charge of e) and that the distance between them varies. The repulsive Coulomb force the powerful particulars exert on each other varies in accordance with Coulomb law. There might be some uninstantiated distances between two powerful particulars having the charge of e. However, we have clear reason to maintain that repulsive force varies in accordance with the inverse square law: the respective causal process (and electro-magnetic field) spreads evenly in a three-dimensional space.

By contrast, we lack similar means of assessing the truth of the counterfactual about the behaviour trope $t_{1}$. Unless we can find a clear method of assessing the truth of such counterfactual claims as instances of the determinate law about the behaviour $t_{1}$, I remain sceptical of whether the corresponding determinate laws are true.

The present approach explains why the dispositional tropes and causal processes can take care of the governing function, i.e., determine that the resulting forces accord with the functional formula. By contrast, we lack similar means of dealing with the guaranteeing function, i.e., to secure that the functional law is true of every possible value of the quantities it contains. Since the causal laws of action are not entities but rather statements about the behaviour of objects (and their constituent tropes) and the resulting causal processes, I do not consider this as a serious defect. Although all tropes falling under a certain determinable and the causal processes they produce act in accordance with a functional law, there might still be limitations in the domain of application of the law: the kinds of possible properties of which it is true.

\section{Conclusion}

Starting with the assumption that all fundamental property tropes are dispositional, I have argued in the present paper that property tropes and the causal processes they produce can, in relevant part, account for the truth of the causal functional laws. First, tropes fall under determinates and determinables because they are connected by the formal relations of proportion. Second, the manifestations of the most dispositional property tropes are causal processes. The forces (e.g., gravitational force, Coulomb force) resulting from these causal processes are connected by the proportion relations in accordance with the proportion 
relations between the tropes producing the processes. Hence, property tropes and the respective causal processes suffice to secure that the resulting forces accord with the functional formula. While we seem to be able to account for the truth of the functional formula for every determinate value of existent causal powers, this bottom-up approach gives no guarantee that the functional law is true of every of its possible but uninstantiated values.

\section{References}

Armstrong, D.M. (1997): A World of States of Affairs, (Cambridge: Cambridge University Press).

Bigelow, J. \& Pargetter, R. (1990): Science and Necessity, (Cambridge: Cambridge University Press).

Bird, A (2007): Nature's Metaphysics: Laws and Properties, (Oxford: Oxford University Press).

Campbell, K. K. (1990): Abstract Particulars, (Oxford: Basil Blackwell).

Denkel, A. (1996): Object and Property, (Cambridge: Cambridge University Press).

Ehring. D. (2011): Tropes: Properties, Objects, and Mental Causation, (Oxford: Oxford University Press).

Ellis, B. (2001): Scientific Essentialism, (Cambridge: Cambridge University Press).

Ellis, B. \& Lierse, C. (1994): "Dispositional Essentialism", Australasian Journal of Philosophy 72, 27-45.

Griffiths, D. (1987): Introduction to Elementary Particles, (New York: John Wiley \& Sons).

Handfield, T. (2010): "Dispositions, Manifestations, and Causal Structure" in Anna Marmodoro (ed.), The Metaphysics of Powers: Their Grounding and Their Manifestations, (London: Routledge), pp. 106-132.

Hendry, R. and Rowbottom, D. (2009): "Dispositional essentialism and the necessity of laws", Analysis 69, 668-677.

Keinänen, M. (2011): “Tropes - the Basic Constituents of Powerful Particulars?", Dialectica 65: 3, 419-450.

Keinänen, M. \& Hakkarainen, J. (2013): “The Problem of Trope Individuation: A Reply to Lowe", forthcoming in Erkenntnis.

Keller, P. \& Correia, F. (2004): "Introduction", Dialectica 58, 3, 275-278.

Lowe, E.J. (1998): The Possibility of Metaphysics - Substance, Identity and Time, (Oxford: Clarendon Press).

Lowe, E.J. (2006): The Four-Category Ontology, (Oxford: Oxford University Press).

Lowe, E.J. (2009): More Kinds of Being, (Oxford: Wiley-Blackwell).

Massin, O. (2009): “The Metaphysics of Forces”, Dialectica 64 (4), 555-589.

Maurin, A-S. (2002): If Tropes, (Dordrecht: Kluwer Academic Publishers).

Morganti, M. (2009): “Tropes and Physics”, Grazer Philosophische Studien 78, 185205.

Mulligan, K.; Simons, P.M. \& Smith, B. (1984): "Truthmakers", Philosophy and Phenomenological Research XIV: 3, 278-321.

Mumford. S. (2004): Laws in Nature, (London: Routledge).

Mumford, S. (2006): “The Ungrounded Argument”, Synthese 149, 471-489. 
Schaffer, J. (2001): “The Individuation of Tropes". Australasian Journal of Philosophy 79, 2, 147-157.

Simons, P. M. (1987): Parts - a Study in Ontology (Oxford: Clarendon Press).

Simons, P. M. (1994): "Particulars in Particular Clothing - Three Trope Theories of Substance", Philosophy and Phenomenological Research LIV: 3, 553-75.

Simons, P. (2003): "Tropes, Relational", Conceptus 35, 53-73.

Smith, B. (1998): "The Basic Tools of Formal Ontology", in Guarino, N. (ed.): Formal Ontology in Information Systems, (Amsterdam: IOS Press).

Smith, B \& Mulligan, K. (1983): "Framework for Formal Ontology", Topoi 2, 73-85.

Williams, D. C. (1953): “On the Elements of Being I", Review of Metaphysics 7, 3-18.

Williams, D. C. (1986): "Universals and Existents", Australasian Journal of Philosophy 64, 1, 1-14.

Yates, D. (2012): "The Essence of Dispositional Essentialism", Philosophy and Phenomenological Research, forthcoming. 